\title{
THE EFFECT OF ENTRAINMENT SITE AND INSPIRATORY PRESSURE ON THE DELIVERY OF OXYGEN THERAPY DURING NON-INVASIVE MECHANICAL VENTILATION (NIMV) IN ACUTE COPD PATIENTS
}

\author{
Sundeep Kaul, MRCP*, lan M. Stell, MD, MRCP*, S. Chinn, PhD*, M. Polkey, PhD, MRCP\# and J. Moxham, MD, MRCP* \\ *Dept Respiratory Medicine, Kings College Hospital, London, United Kingdom \\ \#Respiratory Medicine, Royal Brompton Hospital, London, United Kingdom
}

WINNING ABSTRACT: Supplemental $\mathrm{O}_{2}$ is frequently added to bi-level non-invasive ventilation circuits to maintain $\mathrm{Sa}, 02>90 \%$. Oxygen can be added at several points \& in the presence of different inspiratory pressures. The effect of varying entrainment sites and inspiratory pressures (IPAP) on PO2, PCO2, Fio2, inspiratory triggering and expiratory triggering in COPD patients is unknown.

18 patients with stable COPD (mean FEV1 47\%) participated in the study. Oxygen was added at 4 sites in the ventilatory circuit (site 1: between mask and exhalation port; site 2: just distal to exhalation port; site 3: at ventilator outlet; site 4: directly into the mask via an inlet). The effect of varying entrainment sites and inspiratory pressures on arterial PO2, PCO2, FIO2, was recorded at 3 mins. The same full face mask (Respironics, Image 3) \& ventilator (Respironics, BIPAP ST 30) was used.

Results for PO2 are shown at IPAP 10/EPAP 4 [table 1].

Anova was used to analyse the data.

Site 4 (via mask) was associated with a significantly higher PO2 at all flow rates compared with sites 1, 2 and 3 (P<0.001).

Site 3 (at ventilator outlet) was associated with the lowest PO2 at all flow rates, particularly at $15 \mathrm{~L} \mathrm{~min}(\mathrm{P}<0.001)$.

Adding oxygen to the mask at lower IPAPs result in higher oxygen delivery.

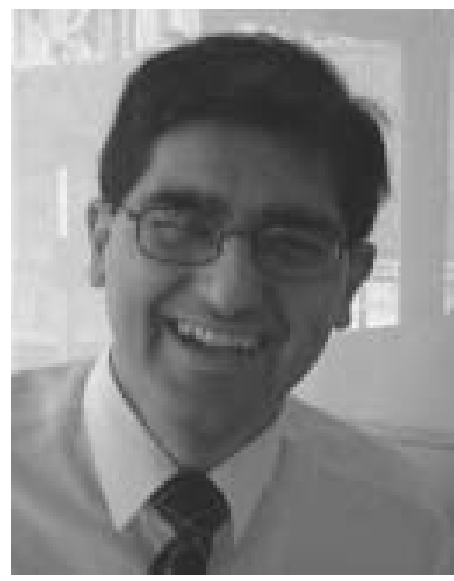

Sundeep Kaul

Dept of Asthma, Allergy and Respiratory Science, Kings College London School of Medicine, Kings College Hospital, London, UK

\section{MY JOB AND THE UNIT IN WHICH I WORK}

I work as a Clinical Research Fellow in the Muscle Laboratory, in the Dept of Respiratory Medicine, Kings College Hospital, London, UK. The Muscle Laboratory at Kings College Hospital has provided a national service for the assessment of respiratory muscle function for the past $20 \mathrm{yrs}$ under the leadership of Prof. John Moxham.

The laboratory at Kings College Hospital is able to offer a onestop service whereby patients can have their lung function, blood gases, muscle function tests and functional capacity assessed at one centre and on one visit.
The unit specialises in performing and interpreting respiratory and nonrespiratory muscle function tests. Tests of inspiratory and expiratory muscle strength are divided into simple volitional tests performed by the patient and nonvolitional tests involving stimulation of the phrenic nerves, which supply the diaphragm. Each set of tests can be subdivided into either noninvasive, measuring pressure at the mouth or in the nose, or semi-invasive, requiring the passing of balloon catheters down the oesophagus to allow intrathoracic and intra-abdominal pressure measurements. By performing these measurements, we can obtain an accurate assessment of not only global respiratory muscle strength but also diaphragm strength.

The volitional tests are generally simple to perform and provide a good measure of strength, but are limited as patient cooperation and motivation can influence the results. Nonvolitional tests are often useful as accurate, reproducible results can be obtained irrespective of patient cooperation. A standard assessment involves both volitional and nonvolitional assessments.

This service is unique in that equipment, expertise and personnel are available to perform assessments across the age range, from children to adults. Although some tests are more suited to adults than children, a full assessment of respiratory and peripheral muscle function can be performed in all age groups.

The department has a long history of research in areas allied to respiratory muscle physiology, particularly noninvasive ventilation (NIV), pulmonary rehabilitation, sleep disorders, neuromuscular disease and chronic obstructive pulmonary disease (COPD). My role includes managing the clinical referrals and carrying out research in NIV in patients with COPD. 
TABLE 1 Inspiratory positive airway pressure 10/expiratory positive airway pressure 4

\begin{tabular}{|c|c|c|c|c|}
\hline $\begin{array}{l}\text { Oxygen flow rate in } \\
L \cdot \min ^{-1}\end{array}$ & $\mathrm{PO}_{2}$ at site 1 & $\mathrm{PO}_{2}$ at site 2 & $\mathrm{PO}_{2}$ at site 3 & $\mathrm{PO}_{2}$ at site 4 \\
\hline 5 & $5.92 \pm 2.02$ & $6.76 \pm 1.94$ & $5.02 \pm 1.61$ & $10.3 \pm 1.93$ \\
\hline 15 & $18.31 \pm 5.80$ & $17.46 \pm 5.65$ & $10.81 \pm 2.62$ & $26.03 \pm 5.69$ \\
\hline
\end{tabular}

Data are presented as mean $\pm \mathrm{SD}$. $\mathrm{PO}_{2}$ : oxygen arterial pressure.

\section{MY WINNING POSTER AS PART OF MY RESEARCH}

My PhD is entitled "Improving the patient ventilator interaction during noninvasive ventilation". It is well recognised that impaired patient-ventilator interaction reduces the compliance of patients and the therapeutic benefit of NIV.

The aim of this work was to study the factors that influence the patient-ventilator experience during NIV, with a view to improving its successful application. This in turn could mean more patients successfully tolerating NIV for longer periods of time and, therefore, benefitting from this intervention. This particular part of the research focussed on the way in which oxygen is delivered during NIV.

The effect of oxygen flow rates on ventilator function and on gas concentrations ultimately breathed in by patients are unknown. Therefore, we set out to ascertain how we can deliver adequate oxygen concentrations without losing ventilator-patient coordination, and to understand the limits to oxygen delivery with standard equipment.

This study was undertaken in stable COPD patients in the laboratory. The effect of varying oxygen entrainment rate, site of oxygen entrainment and ventilator inspiratory pressure were measured in the following: 1) mask oxygen concentration; 2) ventilator inspiratory trigger time; 3) ventilator inspiratory duration; 4) transcutaneous oxygen; and 5) carbon dioxide tensions. Mask oxygen concentration was measured using a fast-responding paramagnetic oxygen sensor; other variables were measured using standard techniques. The measured variables allow calculation of ventilation/perfusion ratio matching, an important guide to whether increased inspired oxygen concentration will relieve hypoxaemia.

\section{MY RESEARCH AS PART OF MY WORKING GROUP/ RESEARCH TEAM}

This fits well into the main research area of our unit. I am part of a wider team from the Respiratory Muscle Laboratory, led by Prof. John Moxham, which currently comprises three undergraduates, five Clinical Research Fellows and one
Senior Lecturer in Respiratory Physiology. Prof. Moxham's research is focussed on the respiratory muscle pump and the interactions of ventilatory load, ventilatory capacity and neural respiratory drive. Research into NIV, which reduces ventilatory load and respiratory drive, has been an important research area. The group reported the first controlled clinical trial of NIV in acute exacerbation of COPD and have demonstrated that NIV can facilitate exercise training in COPD. Tolerance of NIV in COPD is an important challenge, as is the optimisation of oxygen therapy during ventilation. The current study contributes to the overall research programme of the group.

\section{THE IMPACT OF MY WORK ON CLINICAL OR RESEARCH PRACTICE}

The majority of units in the UK use flow-triggered and cycled, pressure-targeted bi-level ventilators to deliver NIV. These devices compensate well for leaks but do not typically have an oxygen control. These NIV ventilators entrain room air and oxygen enrichment requires supplemental oxygen to be fed either proximally into the circuit or directly into the mask. Oxygen is added to the circuit at unspecified points using varied flow rates; however, the exact inspired concentration of oxygen is generally unknown. Therefore, there is a lack of data on the oxygen concentration that is delivered by commonly used ventilators. Furthermore, the effect of adding oxygen to the circuit on ventilator triggering and cycling is largely unexplored.

The data from this study will help clinicians to maximise oxygen delivery during NIV and provide predictable oxygen concentrations without interfering with ventilator triggering or cycling.

Furthermore, if the patient's hypoxaemia fails to improve despite optimising the technical parameters, then this serves as a good indicator to the clinician as to the severity of the patient's condition and has implications for further management. 